Santé mentale au Québec

Analyse des coûts et de l'efficacité d'une psychothérapie pour le Trouble panique avec agoraphobie versus un traitement combinant la pharmacothérapie et la psychothérapie

Analysis of the cost and the effectiveness of a psychotherapy for panic disorder with agoraphobia (PDA) versus a treatment combining pharmacotherapy and psychotherapy

Análisis de costos y de la eficacia de una psicoterapia para el trastorno de pánico con agorafobia en comparación con un tratamiento que combina la farmacoterapia y la psicoterapia.

Análise dos custos e da eficácia de uma psicoterapia para o Transtorno do Pânico com Agorafobia em comparação com um tratamento que associa a farmacoterapia e a psicoterapia

André Marchand, Vanessa Germain, Daniel Reinharz, Nicole Mainguy et Pierre Landry

Volume 29, numéro 2, automne 2004

Les psychoses réfractaires : modèles de traitement américains et européens

URI : https://id.erudit.org/iderudit/010837ar

DOI : https://doi.org/10.7202/010837ar

\section{Aller au sommaire du numéro}

Éditeur(s)

Revue Santé mentale au Québec

\section{ISSN}

0383-6320 (imprimé)

1708-3923 (numérique)

Découvrir la revue

\section{Citer cet article}

Marchand, A., Germain, V., Reinharz, D., Mainguy, N. \& Landry, P. (2004). Analyse des coûts et de l'efficacité d'une psychothérapie pour le Trouble panique avec agoraphobie versus un traitement combinant la pharmacothérapie et la psychothérapie. Santé mentale au Québec, 29(2), 201-220. https://doi.org/10.7202/010837ar
Résumé de l'article

La pharmacothérapie et la thérapie cognitive-comportementale (TCC) sont des traitements de choix pour le Trouble panique avec agoraphobie (TPA) mais demeurent généralement dispendieux. La présente étude vise à comparer les ratios coûts/efficacité d'une TCC pour le TPA versus un traitement combinant la TCC à une pharmacothérapie. Quatre-vingt-quatre participants suivent une TCC combinée ou non avec une médication. Ils sont évalués avant et après le traitement ainsi qu'au suivi d'un an et de deux ans. L'efficacité de la thérapie est calculée à partir d'un "Index de fonctionnement global " pour chaque temps de mesure. Les coûts directs et indirects pour leur part, sont calculés grâce à une " Entrevue sur les coûts de la santé ». Les résultats suggèrent que pour une efficacité équivalente, la TCC seule demeure moins dispendieuse qu'une TCC combinée à une pharmacothérapie. Elle offre donc un meilleur ratio coûts/efficacité. Ce ratio tend même à augmenter avec le temps. 


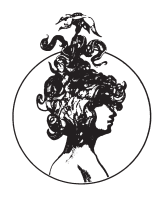

\title{
Analyse des coûts et de l'efficacité d'une psychothérapie pour le Trouble panique avec agoraphobie versus un traitement combinant la pharmacothérapie et la psychothérapie
}

\author{
André Marchand* \\ Vanessa Germain** \\ Daniel Reinharz ${ }^{* * *}$ \\ Nicole Mainguy ${ }^{* * * *}$

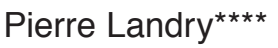

La pharmacothérapie et la thérapie cognitive-comportementale (TCC) sont des traitements de choix pour le Trouble panique avec agoraphobie (TPA) mais demeurent généralement dispendieux. La présente étude vise à comparer les ratios coûts/efficacité d'une TCC pour le TPA versus un traitement combinant la TCC à une pharmacothérapie. Quatre-vingt-quatre participants suivent une TCC combinée ou non avec une médication. Ils sont évalués avant et après le traitement ainsi qu'au suivi d'un an et de deux ans. L'efficacité de la thérapie est calculée à partir d'un «Index de fonctionnement global» pour chaque temps de mesure. Les coûts directs et indirects pour leur part, sont calculés grâce à une «Entrevue sur les coûts de la santé ». Les résultats suggèrent que pour une efficacité équivalente, la TCC seule demeure moins dispendieuse qu'une TCC combinée à une pharmacothérapie. Elle offre donc un meilleur ratio coûts/efficacité. Ce ratio tend même à augmenter avec le temps.

\footnotetext{
* $\quad$ Ph.D., Professeur à l'Université du Québec à Montréal et chercheur au Centre de recherche Fernand- Seguin.

** Étudiante au doctorat en psychologie à l'Université du Québec à Montréal.

*** MD, Chercheur au Centre hospitalier universitaire de Québec et professeur à l'Université Laval.

**** Ph.D., MD, Clinicien et chercheur à l'Hôpital Louis. H. Lafontaine.

\section{Remerciements}

Cette étude a été rendue possible grâce à une subvention du Conseil québécois de la recherche sociale (CQRS) (RS-3166), grâce à une bourse du FCAR décernée au deuxième auteur ainsi que du support constant du Centre de recherche Fernand-Seguin, de la Clinique d'intervention cognitivo-comportementale de l'Hôpital Louis-H. Lafontaine, la Clinique des troubles anxieux de l'Hôpital du Sacré-Cour ainsi que l'Université du Québec à Montréal. Les auteurs remercient également les psychologues et psychiatres qui ont bien voulu participer à l'évaluation et au traitement des participants de l'étude.
} 
e trouble panique avec agoraphobie (TPA) ou sans agoraphobie (TPSA) est une problématique qui touche entre $2,5 \%$ et $6 \%$ de la population (Kessler et al., 1994). Parmi les différents troubles anxieux, le TPA demeure celui qui a fait l'objet du plus grand nombre d'études contrôlées (Antony et Swinson, 1996). L'efficacité des différentes approches pour traiter ce trouble a maintes fois été démontrée. Les interventions pharmacologiques et particulièrement les thérapies cognitive-comportementales (TCC) constituent des traitements de premier choix pour ce trouble (Craske et al., 2000; Wolfe et Maser, 1994).

En ce qui a trait à la pharmacothérapie, plusieurs médicaments se sont révélés plus efficaces qu'un placebo pour traiter le TPA notamment les benzodiazépines, les antidépresseurs tricycliques et les ISRS (Antony et Swinson, 1996). La majorité des études sur le sujet ont démontré que la prise de médication permet de diminuer la fréquence des attaques de panique, l'anxiété anticipatoire, l'évitement, la dépression associée au trouble en plus d'augmenter le niveau de fonctionnement global (Gorman et al., 1998). Toutefois, l'utilisation de la pharmacothérapie peut présenter différents problèmes tels que l'apparition d'effets secondaires non désirés, un certain risque de dépendance aux médicaments ainsi qu'une fréquence relativement élevée de différents symptômes de rebond et de sevrage menant à un taux de rechute important (Wolfe et Maser, 1994).

La TCC, pour sa part, constitue la seule forme de psychothérapie ayant une efficacité clairement démontrée pour ce trouble (Craskeet al., 2000 ; Marchand et Boivin, 1999). Cette intervention s'avère plus efficace que l'absence de traitement ou des interventions psychologiques non spécifiques (par exemple une psychothérapie de soutien) et plus efficace que certains traitements pharmacologiques (Barlow et al., 2000). Entre $80 \%$ et $95 \%$ des clients obtiennent une amélioration clinique significative et atteignent un niveau de fonctionnement tout à fait satisfaisant. En fait, après trois mois de traitement, les clients peuvent être complètement libérés de leurs attaques de panique, de leurs comportements d'évitement et de leur détresse psychologique (Marchand et Boivin, 1999). On note aussi que ce type de thérapie est bien toléré et que les taux d'abandon sont faibles (5-6\%) (Roth et Fonagy, 1996). Par ailleurs, des suivis effectués dans divers pays permettent de constater que les effets thérapeutiques se maintiennent à très long terme, ce qui présente un avantage sur l'approche pharmacologique pour laquelle des rechutes surviennent généralement suite à la cessation de la médication (Clum, 1989). 
Actuellement, plusieurs professionnels de la santé optent pour l'association de la pharmacothérapie et de la TCC. De nombreuses études ont donc été menées afin de comparer l'efficacité de ces trois différentes approches (TCC, pharmacothérapie et combinaison des deux traitements). La recension des écrits menée par Antony et Swinson (1996) a démontré que, dans plusieurs études, la TCC est apparue plus efficace que l'approche pharmacologique, particulièrement à long terme. Dans d'autres études, les différences observées sont minimes. Deux études seulement ont démontré que la pharmacothérapie donne de meilleurs résultats que la TCC. Par ailleurs, de manière générale, le traitement combiné ne se révèle pas plus efficace que chacun des traitements administrés isolément (Antony et Swinson, 1996, 34). Une méta-analyse comparant 43 études de traitement et réalisée par Gould et al. (1995) permet d'observer une taille d'effet plus élevée pour la TCC avec exposition aux stimuli intéroceptifs $(.88)$ que pour le traitement pharmacologique (.47) ou la combinaison des deux traitements (.56). Les taux d'abandon constatés dans cette méta-analyse vont dans le même sens et sont respectivement de 5,6\%,19,8\% et $22 \%$ (Gould et al., 1995). Il semble donc que la TCC offre une efficacité légèrement supérieure à la pharmacothérapie et à la combinaison des deux.

Toutefois, certaines réserves doivent être prises en considération lors de l'interprétation de ces résultats. Tout d'abord, plusieurs études portant sur l'efficacité de la TCC dans le traitement du TPA excluent les clients aux prises avec une agoraphobie élevée et donc, surestiment possiblement cette efficacité. Les études controlées incluant des clients ayant un large spectre d'évitement agoraphobique obtiennent en effet des taux d'attrition plus élevés ainsi qu'un plus faible pourcentage d'amélioration clinique notable au post-test et au suivi. Par ailleurs, la rigueur méthodologique et les différentes mesures d'efficacité des études comparées demeurent très variables entre elles, ce qui peut expliquer l'inconsistance des résultats obtenus jusqu'à présent (White et Barlow, 2002). Toutefois, bien que les résultats à ce sujet semblent encore pour le moins controversés, une certaine tendance voulant que la TCC obtienne de meilleurs résultats et particulièrement à long terme peut être observée. Par contre, très peu d'études tentent de vérifier spécifiquement quelle intervention s'avère la plus efficace entre un traitement combiné et une TCC administrée seule. Ce manque est d'autant plus flagrant en ce qui concerne les individus ayant un TPA d'intensité élevée.

Outre l'efficacité des différentes interventions potentielles pour le TPA, les coûts qui y sont associés demeurent une variable très 
importante à considérer. En effet, bien que les coûts liés à cette problématique soient très élevés (incapacité de travailler, absentéisme au travail, baisse de productivité, utilisation marquée des services de soins de santé, etc.), les traitements, qu'ils soient psychologiques, pharmacologiques ou une combinaison des deux coûtent aussi très chers à l'individu et à la société (Ettigi et al., 1997; Leon et al., 1995; Rees et al., 1998; Siegel et al., 1990). Ainsi, afin de maximiser l'efficience des services offerts, il demeure indispensable de bien évaluer les coûts associés à chaque traitement et particulièrement selon leur efficacité respective. Diverses études ont été menées afin de comparer les ratios coûts/efficacité d'une TCC versus un traitement pharmacologique. À titre d'exemple, les résultats obtenus par Otto et al. (2000) démontrent que la TCC, particulièrement de groupe, présente un meilleur ratio coûts/efficacité que la pharmacothérapie. L'analyse des coûts (dollars U.S., année 1997), qui inclut les coûts directs du traitement, de la médication et des traitements complémentaires, correspond à $2305 \$$ pour la pharmacologie, $1357 \$$ pour la TCC conventionnelle et $523 \$$ pour la TCC de groupe. Le rapport coûts/efficacité à quatre mois indique un coût de $246 \$$ pour la TCC de groupe, de $565 \$$ pour la TCC individuelle et de $447 \$$ pour la pharmacothérapie par point d'amélioration (Otto et al., 2000). Par ailleurs, la méta-analyse menée par Gould et al. (1995) visant à comparer l'efficacité des études avec groupe contrôle entre la TCC, la pharmacothérapie et les traitements combinés du TPA, indique une estimation des coûts directs moyens d'un traitement typique sur une période de deux ans, soit $1650 \$$ pour la TCC individuelle, $840 \$$ pour la TCC de groupe, $1800 \$$ pour l'alprazolam à $2.5 \mathrm{mg}, 3312 \$$ pour l'alprazolam à $6 \mathrm{mg}, 912 \$$ pour l'imipramine et $3504 \$$ pour la fluoxetine (Gould et al., 1995). Toutefois, cette méta-analyse ne constitue pas une évaluation coûts/efficacité des traitements du trouble panique. Ainsi, bien que ces résultats offrent une estimation intéressante des coûts des traitements en fonction de leur efficacité globale, elle ne permet aucunement de tirer des conclusions quant aux ratios coûts/efficacité de ces traitements.

À notre connaissance, bien que la TCC semble à première vue présenter un meilleur ratio coûts/efficacité que les traitements pharmacologiques, aucune étude n'a su comparer les ratios des traitements combinant la TCC à une pharmacothérapie versus la TCC sans médication et encore moins avec des individus ayant un niveau d'agoraphobie élevé. Comme les traitements combinés semblent de plus en plus utilisés, l'examen de cette comparaison paraît essentiel afin de maximiser l'efficience des services offerts pour traiter le TPA. 


\section{Objectifs et hypothèses de la recherche}

Le principal objectif de la présente étude consiste à comparer les ratios coûts/efficacité d'une TCC pour un TPA d'intensité modérée à sévère versus un traitement combinant la TCC à une pharmacothérapie. De plus, l'examen de l'efficacité clinique et statistique des deux conditions de traitement quant à la réduction des symptômes liés au TPA sera effectué. Les hypothèses de recherche sont les suivantes:

1) Les participants des deux conditions de traitement obtiendront une amélioration statistique et clinique significative au niveau des différentes mesures du TPA.

2) De plus, les scores obtenus par les participants des deux conditions de traitement seront supérieurs aux scores des participants de la condition liste d'attente.

3) Finalement, la TCC administrée isolément obtiendra un meilleur ratio coûts/efficacité que le traitement combiné, et ce ratio tendra à se maintenir à plus long terme. Cette hypothèse est avancée considérant les coûts possiblement plus élevés du traitement combiné en raison de la prise de médication ainsi que la plus grande efficacité des traitements psychologiques en ce qui concerne le maintien des gains à long terme.

\section{Méthode}

La présente étude s'inscrit à l'intérieur d'un projet de recherche de plus grande envergure portant sur une évaluation clinique, psychosociale et économique de trois modalités de traitement psychologique $\mathrm{du}$ trouble panique avec agoraphobie, soit un traitement individuel, de groupe ou autogéré (Marchand et al., 2000). Ce dernier projet de recherche a donc permis d'identifier quelle modalité de traitement est à la fois la plus efficace et la moins coûteuse. Les résultats alors obtenus n'ont révélé aucune différence statistique et clinique significative entre les trois modalités de traitement quant à leur efficacité. C'est pourquoi dans la recherche actuelle, aucune différenciation n'est faite quant aux trois modalités de traitement. Les résultats sont donc regroupés et analysés de manière globale.

\section{Procédures}

L'étude est menée auprès de 84 individus diagnostiqués avec un trouble panique avec agoraphobie de niveau modéré à sévère (score de trois ou plus à l'Échelle globale de sévérité des symptômes, Lavallée et Lebeau, 1992; adaptation du Global Assessment of Severity Scale, Mavissakalian et al., 1983). Tous les participants reçoivent un traitement 
cognitif-comportemental pour le TPA. Vingt-sept participants sont sélectionnés aléatoirement pour faire partie d'une condition d'attente qui constitue le groupe contrôle. Ces participants attendent 14 semaines avant de recevoir leur traitement. Parmi les 84 participants de l'étude, 57 prennent une médication de type anxiolytique, antidépresseur ou autres psychotropes avant de débuter la thérapie. Ils ont pour consigne d'en poursuivre l'utilisation pour toute la durée du traitement. Les 27 participants qui ne prennent aucun médicament doivent s'abstenir d'en commencer l'usage. Sur cette base de participants, nous avons donc pu choisir les participants selon qu'ils suivent un traitement combinant la pharmacothérapie et la TCC ou la TCC seule. Seuls les résultats des participants ayant pris une médication de manière stable sont comparés à ceux n'ayant pas de pharmacothérapie.

La TCC est administrée dans deux cliniques spécialisées dans le traitement des troubles anxieux et menée par des psychologues et des psychiatres possédant une grande expertise dans l'évaluation et le traitement des troubles anxieux. L'intégrité du traitement est maintenue par l'utilisation d'un protocole de traitement structuré et adapté (Craske et Barlow, 1993). Toutes les sessions d'intervention sont enregistrées sur bande audio. Un échantillon d'environ $30 \%$ des sessions de traitement est sélectionné aléatoirement et évalué objectivement par des évaluateurs indépendants, aveugles aux objectifs et hypothèses de la recherche, à partir d'une grille d'intégrité thérapeutique conçue pour chaque session (Roberge et Marchand, 2000). La grille d'intégrité thérapeutique permet d'observer un taux d'intégrité moyen des interventions de 96,8 \%. Le protocole de traitement inclut les éléments thérapeutiques suivants : psycho-éducation concernant le trouble et démystification des symptômes de panique, rééducation respiratoire, correction cognitive, exposition aux stimuli intéroceptifs, exposition in vivo et prévention de la rechute. Les participants reçoivent un manuel de style bibliothérapeutique au début du traitement et on leur fournit de la lecture et des exercices à pratiquer.

\section{Évaluations}

Chaque participant doit remplir une série de huit questionnaires avant le début du traitement afin d'obtenir un niveau de base pour chaque variable étudiée. Des évaluations de suivi sont ensuite menées immédiatement après la fin du traitement ainsi qu'un an et deux ans plus tard. Ces questionnaires constituent les principaux évaluateurs de progrès et permettent donc d'évaluer l'efficacité du traitement. En premier lieu, la sévérité de l'évitement phobique des sujets agoraphobes ainsi que la fréquence des attaques de panique au cours de la dernière 
semaine sont mesurées par l'Inventaire de mobilité pour l'agoraphobie (IMA : validation québécoise du Mobility Inventory for Agoraphobics, Chambless et al., 1985; Stephenson et al., 1997). Ensuite, le degré de peur face à différentes sensations physiques généralement associées à l'attaque de panique est mesuré grâce au Questionnaire sur les sensations physiques (QSP: validation québécoise du Body Sensations Questionnaire, Chambless et al., 1984 ; Stephenson et al., 1999). Les pensées catastrophiques reliées aux conséquences de l'attaque de panique sont évaluées à l'aide du Questionnaire sur les pensées phobiques (QPP: validation québécoise de l'Agoraphobic Cognitions Questionnaire, Chambless et al., 1984; Stephenson et al., 1998). Ensuite, les niveaux d'anxiété et de dépression sont évalués par les Inventaires d'anxiété et de dépression de Beck (IAB : validation québécoise du Beck Anxiety Inventory, Beck et al., 1988; Freeston et al., 1994; IDB : validation québécoise du Beck Depression Inventory, Beck et al., 1979; Gauthier et al., 1982). L'Index de sensibilité à l'anxiété (ISA: validation québécoise de l'Anxiety Sensitivity Index, Reiss et McNally, 1985; Stephenson et al., 1996) mesure pour sa part la peur d'un ensemble de symptômes anxieux. Finalement, la sévérité du TPA selon le nombre de sphères de fonctionnement affectées est mesurée à l'aide de l'Échelle globale de sévérité des symptômes (EGSS: Lavallée et Lebeau, 1992). Ces huit questionnaires ont été choisis car ils sont utilisés dans plusieurs recherches antérieures (Barlow, 1988; Clum et al., 1993; Hersen et Bellack, 1988), lors d'une recherche subventionnée par le CQRS (Marchand et al., 2000) ainsi que pour leurs qualités psychométriques.

Par ailleurs, deux entrevues structurées sont aussi administrées, soit l'Entrevue structurée pour les troubles anxieux selon le DSM-IV (ESTA-IV: adaptation québécoise de l'Anxiety Disorders Interview Schedule for DSM-IV, Brown et al., 1994 ; Marchand et Boivin, 1997) ainsi que l'Entrevue sur les coûts de la santé (ECS: Roberge et al., 2000). La première entrevue permet d'établir le diagnostic différentiel alors que la seconde permet entre autre de colliger les médicaments consommés durant les trois mois précédant le début du traitement, leur posologie ainsi que les coûts associés.

\section{Index de fonctionnement global}

Afin de déterminer la valeur thérapeutique de chaque condition de traitement, un index de fonctionnement global est calculé. Cet index s'obtient en comparant le niveau de fonctionnement du participant (sur certaines mesures de la symptomatologie) à la fin du traitement ou lors 
des suivis, avec le niveau de fonctionnement d'une population non clinique. Le calcul de cet index se fait dans un premier temps par le calcul d'un indice d'amélioration cliniquement significatif de type « $\mathrm{C}$ » (Jacobson et Truax, 1992). Cet indice « $\mathrm{C} »$, qui est la valeur optimale que chaque personne doit obtenir lors du post-test et des suivis, permet de juger si l'intervention a produit des changements cliniques significatifs, c'est-à-dire si les participants obtiennent des scores comparables à une population non clinique. Par la suite, pour calculer l'index de fonctionnement global post-traitement, le chercheur constitue un score composite comprenant six mesures définies à priori où chaque participant reçoit 1 point si son résultat est égal ou inférieur à la valeur de l'indice $« \mathrm{C} »$ (niveau fonctionnel) au post-traitement et à la relance. Par contre, si son score est supérieur à la valeur de l'indice « $\mathrm{C} »$, il ne reçoit aucun point (niveau dysfonctionnel). L'index de fonctionnement global peut prendre des valeurs entre 0 et 6 . Finalement un nombre de points optimal que la personne doit obtenir est fixé afin de juger si l'intervention a produit chez le participant un niveau de changement clinique significatif. Dans ce cas-ci, le participant doit obtenir au moins 5 points sur 6 afin d'être jugé «hautement amélioré », 3 ou 4 points pour être jugé «modérément amélioré » et entre 1 et 2 points pour le classer dans la catégorie «légèrement amélioré». Cet index de fonctionnement global de l'individu est nécessaire afin de déterminer si le traitement se révèle efficace ou non. Il s'agit de notre variable dépendante.

\section{Résultats}

\section{Analyses préliminaires}

En ce qui a trait aux données socio-démographiques, des tests $t$ pour échantillons indépendants (âge, nombre d'années de scolarité et nombre d'années avec le TPA) et des tests non paramétriques Khi-carré (sexe et statut civil) sont réalisés afin de comparer l'équivalence des participants entre chacune des deux conditions de traitement (avec médication et sans médication). Les résultats indiquent que les deux groupes ne diffèrent pas de façon significative pour les cinq variables socio-démographiques. Le tableau 1 présente les caractéristiques sociodémographiques des participants des deux conditions. Par ailleurs, des ANOVAS à mesures répétées démontrent qu'il n'y a pas de différence statistique entre les scores des participants de la liste d'attente au moment où ils doivent attendre leur traitement et le pré-test. Finalement, au pré-test, aucune différence n'existe entre les scores des participants de la liste d'attente et les scores de ceux qui n'étaient pas sur la liste d'attente. 
Tableau 1

\section{Caractéristiques socio-démographiques des participants selon les conditions de traitement avec médication et sans médication}

\begin{tabular}{|l|r|r|}
\hline $\begin{array}{l}\text { Caractéristiques } \\
\text { socio-démographiques }\end{array}$ & $\begin{array}{l}|l| \\
\text { Avec médication } \\
(\mathrm{n}=57)\end{array}$ & $\begin{array}{l}\text { Sans médication } \\
(\mathrm{n}=27)\end{array}$ \\
\hline Âge moyen (Écart-Type) & $39,60(8,47)$ & $38,41(9,05)$ \\
\hline Sexe (\%) & $48(74,07 \%)$ & $20(84,21 \%)$ \\
$\quad$ Féminin & $9(25,93 \%)$ & $7(15,79 \%)$ \\
Masculin & $10(17,54 \%)$ & $7(25,93 \%)$ \\
\hline Statut civil (\%) & $19(33,33 \%)$ & $8(33,33 \%)$ \\
Célibataire & $19(33,33 \%)$ & $3(11,11 \%)$ \\
Union de fait & $8(14,04 \%)$ & $0(0 \%)$ \\
Marié(e) & $1(1,75 \%)$ & $12,85(3)$ \\
Séparé(e) ou divorcé(e) & $12,16(2,62)$ & $12,44(9,75)$ \\
Veuf (ve) & & \\
\hline $\begin{array}{l}\text { Nombre d'années de } \\
\text { scolarité }\end{array}$ & $14,37(10,94)$ & \\
\hline $\begin{array}{l}\text { Nombre d'années avec } \\
\text { TPA }\end{array}$ & & \\
\hline
\end{tabular}

\section{Analyses principales}

\section{Efficacité statistique des deux conditions}

L'efficacité des deux conditions est ensuite examinée à l'aide d'ANOVAS à mesures répétées et révèle un effet temps hautement significatif pour chacun des huit instruments de mesure $(\mathrm{p}<.0001)$. Des analyses plus détaillées à l'aide de contrastes permettent d'observer que les scores à chaque instrument de mesure diminuent de façon significative entre le pré-test et le post-test, pour ensuite se stabiliser entre le post-test et les relances d'un an et de deux ans. Ces analyses permettent de voir que le traitement se révèle efficace pour les deux conditions de traitement et que les gains se maintiennent à long terme. Le tableau 2 présente les moyennes et les écarts-types au pré-test, post-test, relance d'un an et de deux ans pour chacun des instruments de mesure. De plus, les analyses révèlent que les deux traitements obtiennent des résultats significativement supérieurs à la condition liste d'attente. 
Tableau 2

Moyennes et écart-types (É-T) des deux conditions de traitement pour chacune des mesures aux quatres périodes d'évaluation

\begin{tabular}{|l|l|l|l|l|l|}
\hline & & \multicolumn{4}{|c|}{ Périodes d'évaluation } \\
\hline Questionnaires & $\begin{array}{l}\text { Conditions } \\
\text { de traitement }\end{array}$ & $\begin{array}{l}\text { Pré-test } \\
\text { Moy. (E-T) }\end{array}$ & $\begin{array}{l}\text { Post-test } \\
\text { Moy. (E-T) }\end{array}$ & $\begin{array}{l}\text { Rel. 1 an } \\
\text { Moy. (E-T) }\end{array}$ & $\begin{array}{l}\text { Rel. 2 ans } \\
\text { Moy. (E-T) }\end{array}$ \\
\hline \multirow{2}{*}{ IMA seul } & Avec médic. & $3,7(0,8)$ & $2,1(0,7)$ & $2,1(1)$ & $2,2(1)$ \\
& Sans médic. & $3,2(0,8)$ & $1,9(0,8)$ & $1,7(0,7)$ & $1,7(0,7)$ \\
\hline \multirow{2}{*}{ IMA att. panique } & Avec médic. & $3,1(4,2)$ & $0,4(1,1)$ & $1,2(4,2)$ & $1,1(4,2)$ \\
& Sans médic. & $1,8(3)$ & $0,1(0,3)$ & $0,4(0,7)$ & $0,3(0,7)$ \\
\hline \multirow{2}{*}{ QPP } & Avec médic. & $3,3(0,7)$ & $2(0,6)$ & $1,8(0,7)$ & $1,9(0,6)$ \\
& Sans médic. & $2,8(0,8)$ & $1,7(0,7)$ & $1,7(0,8)$ & $1,6(0,7)$ \\
\hline \multirow{2}{*}{ ISA } & Avec médic. & $2,7(0,6)$ & $1,8(0,5)$ & $1,7(0,7)$ & $1,7(0,7)$ \\
& Sans médic. & $2,5(0,5)$ & $1,6(0,5)$ & $1,5(0,5)$ & $1,5(0,5)$ \\
\hline \multirow{2}{*}{ IDB } & Avec médic. & $35,5(9,5)$ & $17,9(9,6)$ & $17,4(12,7)$ & $17,8(11,6)$ \\
& Sans médic. & $28,1(10,5)$ & $13,3(9,5)$ & $13,5(11,3)$ & $12,1(11,8)$ \\
\hline \multirow{2}{*}{ IAB } & Avec médic. & $18,1(9,5)$ & $10,1(8,9)$ & $10,4(9,7)$ & $9,7(9,7)$ \\
& Sans médic. & $12,7(8,8)$ & $6,7(7)$ & $7,6(9)$ & $5,9(6,6)$ \\
\hline \multirow{2}{*}{ EGSS } & Avec médic. & $29,8(12,2)$ & $13,4(10,5)$ & $14,2(12,7)$ & $14,5(13)$ \\
& Sans médic. & $22(16,1)$ & $9,5(7,6)$ & $9,9(11,3)$ & $8,4(10,5)$ \\
\hline & Avec médic. & $5,2(1)$ & $3(1,1)$ & $2,6(1,5)$ & $3(1,4)$ \\
& Sans médic. & $4,8(0,7)$ & $2,7(1,3)$ & $2,1(1)$ & $2,2(1)$ \\
\hline
\end{tabular}

Efficacité différentielle entre les conditions

Afin de vérifier quelle condition s'avère être la plus efficace, des ANCOVAS à mesures répétées ( 2 conditions $X 3$ temps de mesure) sont utilisées. Tout d'abord, immédiatement après la fin du traitement (posttest), on ne remarque aucune différence significative entre les participants qui prennent une médication et ceux qui n'en prennent pas, et ce pour les huit instruments de mesure. Les résultats obtenus sont les suivants : IMA avec attaques de panique $(F(1,76)=0.16, p=.6943)$; IMA seul $(F(1,80)=0.99, p=.3240) ; \operatorname{BAI}(F(1,78)=1.72, p=.1931)$; BDI $(F(1,26)=0.62, p=.4393)$; QPP $(F(1,80)=0.20, p=.6592)$; $\operatorname{QSP}(F(1,80)=1.04, p=.3120) ; \operatorname{EGSS}(F(1,80)=2.17, p=.1444)$; ISA $(F(1,27)=0.11, p=.7448)$. Par ailleurs, aux relances d'un an et de deux ans, on constate qu'il n'y a toujours pas de différence significative entre les participants des deux conditions pour l'ensemble des instruments de mesure. 


\section{Index de fonctionnement global (IFG)}

Ensuite, l'index de fonctionnement global est calculé pour les deux conditions et pour chaque temps de mesure. L'analyse des résultats permet d'observer qu'aucune différence n'est significative au post-test et à la relance d'un an. Toutefois, à la relance de deux ans, on constate une différence significative entre les deux conditions $(F(1,82)=7.27$, $p=.0085$ ). Cette différence suggère qu'à très long terme, les participants de la condition sans médication obtiennent un indice de fonctionnement supérieur à la condition avec médication. Ainsi, bien que les participants des deux conditions tendent à s'améliorer, cette amélioration s'accentue davantage au niveau de la condition sans médication. Le tableau 3 présente les scores moyens pour les deux conditions de traitement au posttest, à la relance d'un an et à celle de deux ans.

\section{Tableau 3}

\section{Index de fonctionnement global moyen au post-test, aux relances d'un an et de deux ans pour les deux conditions de traitement}

\begin{tabular}{|l|l|l|l|}
\hline \multirow{2}{*}{$\begin{array}{l}\text { Conditions de } \\
\text { traitement }\end{array}$} & \multicolumn{3}{|l|}{ Index de fonctionnement global moyen } \\
\cline { 2 - 4 } & Post-test & Un an & Deux ans \\
\hline Avec médication & 3,95 & 4,21 & 3,98 \\
\hline Sans médication & 4,56 & 4,78 & 5,04 \\
\hline
\end{tabular}

\section{Analyses du ratio coûts/efficacité}

\section{Coûts de chaque condition de traitement}

Ensuite, les coûts associés aux deux conditions de traitement sont calculés pour l'ensemble de l'échantillon afin de pouvoir ultimement comparer les ratios coûts/efficacité. En ce qui a trait à la condition avec médication, le calcul inclut notamment les coûts unitaires de chaque médicament de la liste de la RAMQ multipliés par la dose totale consommée au cours de la période d'évaluation. Ces coûts sont rapportés par les participants lors de l'Entrevue sur les coûts de la santé. Seuls les anxiolytiques et les antidépresseurs pris spécifiquement pour traiter les différents symptômes du TPA sont pris en considération. Les frais de pharmacie applicables à chaque mois sont aussi comptabilisés. Ces coûts sont fixés à $7 \$$ pour chaque renouvellement de prescription. Les prix de la médication sont soustraits aux calculs effectués pour la condition sans médication. Par ailleurs, pour les deux conditions, les honoraires des thérapeutes sont calculés. Ils sont moyennés à $75 \$$ par séance de consultation. En effet, selon l'Ordre des psychologues du 
Québec (1999) le salaire d'un psychologue au Québec varie entre 65\$ et $85 \$$. À ces honoraires sont aussi ajoutés des coûts indirects imputables aux frais de transport pour se rendre aux lieux de consultation ainsi qu'au temps durant lequel le participant ne peut poursuivre ses activités quotidiennes en raison des sessions de thérapie. Les frais de transport sont moyennés à $1,85 \$$ par déplacement indépendamment du type de transport utilisé par les participants. Ce coût est fixé selon les tarifs exigés par la Société de Transport de la Communauté Urbaine de Montréal (STCUM). Les coûts liés au temps perdu sont évalués à 6,90\$ par heure de consultation selon le taux horaire en vigueur au Québec. Il est à noter que l'ensemble des coûts (sauf les coûts de la médication) sont essentiellement théoriques, c'est-à-dire qu'ils ne constituent pas des coûts rapportés par les participants mais plutôt des tarifs fixés selon des standards.

Les résultats indiquent des coûts moyens de $787,70 \$$ pour le salaire des thérapeutes, $43,30 \$$ pour les frais de transport ainsi que $145 \$$ pour le temps employé à ne pas pouvoir travailler, et ce pour chaque participant. Le coût moyen du traitement sans médication se situe donc à $976 \$$. En ce qui a trait à la médication, le coût total incluant les prix pour les renouvellements de prescriptions se situe à $83 \$$ en moyenne par client. Ainsi, la condition combinant la TCC avec une médication coûte en moyenne 1059 .

\section{Ratios coûts/efficacité}

La dernière partie de l'analyse des résultats consiste à examiner le rapport coûts/efficacité des deux conditions de traitement au post-test ainsi qu'aux relances d'un an et de deux ans. Tout d'abord, l'efficacité des deux conditions est passée en revue. Enfin, la mise en relation des coûts et de l'efficacité permettra d'obtenir des ratios coûts/efficacité pour les deux conditions de traitement. Les résultats démontrent que les meilleurs ratios proviennent de la condition sans médication, et ce pour tous les temps de mesure. Ce ratio tend d'ailleurs à s'améliorer avec le temps. Le tableau 4 présente les ratios coûts/efficacité des deux conditions de traitement. 
Tableau 4

\section{Rapport coûts/efficacité au post-test et aux suivis d'un an et de deux ans pour les deux conditions de traitement}

\begin{tabular}{|c|c|c|c|c|c|c|}
\hline & \multicolumn{6}{|c|}{ Conditions de traitement } \\
\hline & \multicolumn{3}{|c|}{ Avec médication } & \multicolumn{3}{|c|}{ Sans médication } \\
\hline & Post-test & Un an & Deux ans & Post-test & Un an & Deux ans \\
\hline Coûts & \multicolumn{3}{|l|}{$1059 \$$} & \multicolumn{3}{|l|}{$976 \$$} \\
\hline Efficacité : IFG & 3,95 & 4,21 & 3,98 & 4,56 & 4,78 & 5,04 \\
\hline $\begin{array}{l}\text { Rapport } \\
\text { coûts/efficacité }\end{array}$ & 268,10 & 251,54 & 266,08 & 214,04 & 204,18 & 193,65 \\
\hline
\end{tabular}

\section{Discussion}

La présente étude visait tout d'abord à examiner l'efficacité de deux conditions de traitement différentes pour les individus aux prises avec un TPA d'intensité modérée à élevée. Les deux conditions examinées consistent en une TCC et en un traitement combinant la TCC à une pharmacothérapie. Ces deux traitements ont aussi fait l'objet d'une comparaison avec une condition contrôle constituée d'une liste d'attente. Finalement, l'étude portait sur la comparaison du ratio coûts/efficacité de la TCC versus le traitement combinant la TCC à une pharmacothérapie.

Les résultats de la recherche permettent tout d'abord d'observer que les deux conditions de traitement sont efficaces à court, moyen et long terme pour traiter un TPA d'intensité modérée à sévère et que leur efficacité est comparable statistiquement et cliniquement. Ces résultats appuient donc la première hypothèse de l'étude. De plus, les données démontrent que les deux conditions de traitement obtiennent des résultats supérieurs à la condition contrôle constituée d'une liste d'attente, ce qui confirme la deuxième hypothèse. Toutefois, comme l'indique l'index de fonctionnement global, les participants ayant suivi une TCC sans aucune prise de médication s'améliorent davantage à long terme que les participants de la condition combinée. Ce résultat concorde avec les études qui démontrent un maintien des acquis à long terme pour la TCC seule comparativement à la pharmacothérapie seule où très peu d'études analysent le maintien des acquis à long terme. Différentes raisons peuvent expliquer cette amélioration à long terme, à commencer par un taux de rechute plus élevé chez les individus qui prennent une médication comparativement à ceux qui suivent une TCC seule. Selon plusieurs auteurs, il semblerait que les gens qui attribuent 
l'amélioration de leur état aux médicaments risquent davantage de rechuter que ceux qui l'attribuent aux interventions psychologiques (Antony et Swinson, 1996). De plus, les réactions psychophysiologiques reliées au sevrage de la médication peuvent déclencher la résurgence de différents symptômes de panique chez certains participants.

Toutefois, dans la présente étude, la majorité $(86 \%)$ des participants qui suivaient une pharmacothérapie durant le traitement ont poursuivi leur médication aux suivis d'un an et de deux ans. Ainsi, la moins grande amélioration à long terme des participants de la condition avec médication peut provenir du fait qu'une fois le traitement psychologique terminé, ils ont cessé de faire les exercices appris en thérapie puisque leurs gains sont, selon eux, attribuables aux médicaments. Différentes raisons peuvent par ailleurs expliquer le fait que la majeure partie de l'échantillon ait poursuivi le traitement pharmacologique. Tout d'abord, il est possible qu'ils aient interprété la consigne de maintenir leur médication à un niveau stable durant les 15 semaines de traitement comme un encouragement à poursuivre la pharmacothérapie malgré une diminution marquée de leur symptomatologie. De plus, ils peuvent soit attribuer leur amélioration à la pharmacothérapie ou encore craindre différents symptômes de sevrage suite à la cessation de leur médication. Finalement, il est possible que les participants n'aient pas réévalué leur besoin de poursuivre le traitement pharmacologique malgré que leurs symptômes aient significativement diminué. Considérant le fait que les participants ont souffert du TPA durant en moyenne 14 ans, la prise de médication peut faire partie intégrante de leur vie depuis plusieurs années déjà, bien avant que la présente étude ne débute. Il aurait donc pu être pertinent d'inciter les gens à cesser la pharmacothérapie et d'envisager différentes procédures de sevrage.

En ce qui a trait aux coûts des deux différents traitements, les résultats démontrent que le traitement combiné est légèrement plus dispendieux que la TCC administrée seule. En effet, les coûts de la médication s'ajoutent inévitablement aux coûts associés à la thérapie. Ainsi, pour un niveau d'efficacité comparable, puisque la TCC demeure moins dispendieuse, elle offre un meilleur ratio coûts/efficacité que le traitement combiné. Ce ratio est encore plus avantageux à long terme puisque les gains obtenus chez les participants de la condition sans médication augmentent avec le temps. Ces résultats permettent donc de confirmer la dernière hypothèse voulant que la TCC administrée isolément obtienne un meilleur ratio coûts/efficacité que le traitement combiné.

À la lumière de ces résultats, on peut se demander quel avantage l'ajout d'une médication offre aux individus se voyant administrer une 
TCC pour leur TPA d'une intensité modérée à sévère. Toutefois, avant de conclure que la TCC seule suffit, il pourrait être intéressant d'examiner minutieusement quel type de traitement profite à quel type d'individu. En effet, bien que plus onéreuse, l'ajout de la pharmacothérapie à la TCC peut en outre permettre à certaines personnes d'atteindre un niveau de fonctionnement minimale et nécessaire à l'application de certaines stratégies comportementales et cognitives, notamment lors de l'exposition aux stimulis intéroceptifs et in vivo. Parrallèlement, la TCC peut offrir une aide précieuse afin de permettre aux clients d'abandonner les traitements pharmacologiques sans rechuter. Ainsi, d'autres recherches devront être menées avant de conclure à la supériorité de la TCC seule versus l'ajout d'une médication dans l'application d'une TCC. Étant donné que les résultats des recherches comparant l'efficacité de la thérapie combinée avec l'efficacité d'autres interventions appliquées seules demeurent controversés, il faudrait plutôt orienter les investigations vers l'identification des individus qui peuvent bénéficier d'un traitement combiné. Actuellement, certains chercheurs suggèrent que le traitement combiné peut être utile pour les individus aux prises avec une agoraphobie d'intensité sévère et qui démontrent une réponse incomplète ou partielle à la TCC ou à la pharmacothérapie (Gorman et al., 1998).

Par ailleurs, une limite importante à considérer dans la présente étude concerne le fait que beaucoup plus d'individus prenaient une médication. En effet, 57 participants ont suivi un traitement combiné, alors que 27 ne prenaient aucune médication. Cet écart peut avoir influencé les résultats. Il demeure donc important de conserver certaines réserves à cet égard. De plus, un contrôle rigoureux de la prise de médication des participants demeure difficilement réalisable et peut donc biaiser les données dans le cas où la médication ne serait pas suivie correctement. Dans le même ordre d'idée, l'instrument utilisé pour rapporter le type et la dose de médication utilisée relève de données rétrospectives pouvant entraîner un biais de mémoire de la part des participants. Toutefois, des évaluations à chaque trois mois ont été menées afin de contrer cette limite. Par ailleurs, bien qu'une force de la présente étude ait été de considérer les individus aux prises avec un TPA modéré ou élevé, il aurait pu être intéressant d'inclure aussi les individus ayant un Trouble panique sans agoraphobie ou encore un TPA d'intensité légère. Le taux de rechute et les coûts associés à la médication pourraient être moins élevés et modifier ainsi le ratio du traitement combiné.

En conclusion, la présente recherche contribue au domaine clinique afin de permettre une meilleure utilisation des soins de santé, en 
l'occurrence de la TCC, pour traiter les gens atteints de TPA. Une augmentation de l'accessibilité à des traitements efficaces pour le TPA peut aider à augmenter le niveau de fonctionnement psychosocial des individus atteints en plus de diminuer les coûts déboursés par les clients, les professionnels de la santé et la société. Dans le cadre d'une prochaine recherche, il serait intéressant d'analyser l'impact plus global que peut avoir ces deux types de traitement (TCC vs traitement combiné) sur la réduction de l'ensemble des coûts sociétaux liés au TPA. En effet, le TPA a des répercussions économiques non seulement sur l'individu mais aussi sur la société en général. Ainsi, examiner quel traitement permet la plus grande réduction des coûts sociaux (absentéisme, utilisation marquée des services de santé, etc.) ainsi que la plus grande amélioration de la qualité de vie constitue une analyse fort importante.

\section{Références}

ANTONy, M. M., Swinson, R. P., 1996, Les troubles anxieux et leur traitement : Analyse critique de la littérature sur les traitements fondés sur des preuves, Ministère des Approvisionnements et Services Canada.

BARLOW, D. H., 1988, Anxiety and its disorders: The nature and treatment of anxiety and panic, New York, Guilford Press.

Barlow, D. H., Gorman, J. M., Shear, M. K., Woods, S. W., 2000, Cognitivebehavioral therapy, imipramine, or their combination for panic disorder: A randomized controlled trial, Journal of the American Medical Association, 283, 2529-2536.

Beck, A. T., Epstein, N., Brown, G., Steer, R. A., 1988, An inventory for measuring clinical anxiety: psychometric properties, Journal of Consulting and Clinical Psychology, 56, 893-897.

Beck, A. T., Rush, A. J., Shaw, B. F., Emery, G., 1979, Cognitive Therapy of Depression, New York, Guilford Press.

Brown, T. A., Dinardo, P. A., Barlow, D. H., 1994, Anxiety Disorders Interview Schedule for DSM-IV. Albalny, NY, Graywind Publications.

Chambless, D. L., Caputo, C., Bright, P., Gallagher, R., 1984, Assessment of fear in agoraphobics: The body Sensations Questionnaire and the Agoraphobia Cognitions Questionnaire, Journal of Consulting and Clinical Psychology, 52, 1090-1097.

Chambless, D. L., Caputo, C., Jasin, S., Gracely, E., Williams, C., 1985, The mobility inventory for agoraphobia, Behavior Research and Therapy, 23, $35-44$. 
Clum, G. A., 1989, Psychological interventions vs drugs in the treatment of panic, Behavior Therapy, 20, 429-457.

Clum, G. A., Clum, G. A., Surls, R., 1993, A meta-analysis of treatments for panic disorder, Journal of Consulting and Clinical Psychology, 61, 317-326.

Craske, M. G., Barlow, D. H., 1993, Panic disorder and agoraphobia, in Barlow, D. H., ed., Clinical Handbook of Psychological Disorders, 1-47, New York, Guilford Press.

Craske, M. G., Barlow, D. H., Meadows, E. A., 2000, Mastery of your anxiety and panic: Therapist guide for anxiety, panic and agoraphobia (MAP-3), San Antonio, TX, Graywind/Psychological Corporation.

Ettigi, P., Meyerhoff, A. S., Chirban, J. T., Jacobs, R. J., Wilson, R. R., 1997, The quality of life and employment in panic disorder, Journal of Nervous and Mental Disease, 185, 6, 368-372.

Freeston, M.H., Ladouceur, R., Thibodeau, N., Gagnon, F., Rhéaume, J., 1994, L'inventaire d'anxiété de Beck: propriétés psychométriques d'une traduction française, L'Encéphale, 20, 47-55.

Gauthier, J. G., Morin, C., Thériault, F., Lawson, J. S., 1982, Adaptation française d'une mesure d'auto-évaluation de l'intensité de la dépression, Revue québécoise de psychologie, 3, 13-27.

Gorman, J., Shear, K., Cowley, D., Cross, D., March, J., Roth, W., Shehi, M., 1998, Practice guideline for the treatment of patients with panic disorder, The American Journal of Psychiatry, 155, 5, 1-34.

Gould, R. A., Отto, M. W., Pollack, M. H., 1995, A meta-analysis of treatment outcome for panic disorder, Clinical Psychology Review, 15, 819-844.

Hersen, M., Bellack, A., 1988, Behavioral Assessment: A Practical Handbook, 3rd ed., Elmsford, NY, Pergamon.

JACOBSON, N. S., TruaX, P., 1992, Clinical significance : A statistical approach to defining meaningful change in psychotherapy research, in Kazdin, A. E., ed., Methodological Issues and Strategies in Clinical Research, Washington, American Psychological Association.

Kessler, R. C., Mcgonable, K. A., Zhao, S., Nelson, C. B., Hughes, M., Eshleman, S., Wittchen, H. U., Kendler, K. S., 1994, Lifetime and 12month prevalence of DSM-III-R psychiatric disorders in the United States: Results from the National Comorbidity Survey. Archives of General Psychiatry, 51, 8-19.

LAVAllÉE, K., LeBEAU, J., 1992, Échelle globale de sévérité des symptômes: Adaptation française du Global Assessment of Severity Scale. Montréal : Module de thérapie béhaviorale, Hôpital Louis-H. Lafontaine. 
Leon, A. C., Portera, L., Weissman, M. M., 1995, The social cost of anxiety disorders, British Journal of Psychiatry, 166, 27, 19-22.

Marchand, A., Boivin, I., 1999, Le trouble panique, in Ladouceur, R., Marchand, A., Boisvert, J.M., éds., Les troubles anxieux: approche cognitive et comportementale, Montréal, Édition Gaétan Morin.

Marchand, A., BoIVIN, I., 1997, L'entrevue structurée pour les troubles anxieux selon le DSM-IV, Document inédit.

Marchand, A., Roberge, P., Dupuis, G., Contandriopoulos, A., Mainguy, N., SAVARD, P., 2000, Évaluation clinique, psychosociale et économique de trois modalités de traitement du trouble panique avec agoraphobie, Document inédit, Université du Québec à Montréal, 1-57.

Mavissakalian, M., Michelson, L., Greenwald, D., Komblith, S., GreENwALD, M., 1983, Cognitive-behavioral treatment of agoraphobia: Paradoxical intention vs. self-statement training, Behaviour Research and Therapy, 21, 75-86.

OrdRe Des PSYCHOlOGues Du QuÉBeC, 1999, The Psychologist and You: A guide for people who wish to seek the services of a psychologist (2nd ed.) Brochure, Montréal.

Otтo, M. W., Pollack, M. H., Maki, K. M., 2000, Empirically supported treatments for panic disorder: Costs, benefits, and stepped care, Journal of Consulting and Clinical Psychology, 68, 4, 556-563.

Rees, C. S., Richards, J. C., Smith, L. M., 1998, Medical utilization and costs in panic disorder: A comparison with social phobia, Journal of Anxiety Disorders, 12, 421-435.

ReISS, S., MCNALly, R. J., 1985, The expectancy model for fear, in Reiss, S., et Bootzin, R. R., éds., Theorical Issues in Behavior Therapy, New York, Academic Press.

Roberge, P., Marchand, A., 2000, Grille d'intégrité thérapeutique, Document inédit, Université du Québec à Montréal.

Roberge, P., Marchand, A., Cloutier, K., Reinharz, D., 2000, Entrevue sur les coûts de la santé, Document inédit, Université du Québec à Montréal.

Roth, A., Fonagy, P., 1996, What Works for Whom: A Critical Review of Psychotherapy Research, New York, Guilford.

Siegel, L., Jones, W.C., Wilson, J. O., 1990, Economic and life consequences experienced by a group of individuals with panic disorder, Journal of Anxiety Disorders, 4, 201-211. 
Stephenson, R., Marchand, A., Lavallée, M.-C., 1999, A French Canadian adaptation of the agoraphobic cognitions questionnaire: Cross-cultural validation and gender differences, Scandinavian Journal of Behavior Therapy, 28, 1-12.

Stephenson, R., Marchand, A., Lavallée, M.-C., 1998, Traduction et validation canadienne-française du questionnaire des pensées phobiques, L'Encéphale, 24, 415-425.

Stephenson, R., Marchand, A., LavalléE, M.-C., 1997, Validation de l'Inventaire de mobilité pour l'agoraphobie auprès de la population québécoise francophone, Science et Comportement, 26, 35-38.

Stepenson, R., Marchand, A., Lavallée, M.-C., Brillon, P., 1996, Translation and transcultural validation on the sensibility anxiety index, Résumé du XXVIième International Congress of Psychology (ICP), Montreal, Canada, 31, 3-4, numéro 484-104.

White, K. S., BARlow, D. H., 2002, Panic disorder and agoraphobia, in Barlow, D.H., ed., (Second Edition), Anxiety and its Disorders : The Nature and Treatment of Anxiety and Panic, The Guilford Press, New York.

Wolfe, B. E., Maser, J. D., 1994, Treatment of Panic Disorder : A Consensus Development Conference, American Psychiatric Press, Washington.

\section{ABSTRACT}

\section{Analysis of the cost and the effectiveness of a psychotherapy for panic disorder with agoraphobia (PDA) versus a treatment combining pharmacotherapy and psychotherapy}

Pharmacotherapy and cognitive-behavioral therapy (CBT) are efficient treatments for Panic disorder with agoraphobia (PDA) yet they remain expensive. This study aims to compare the cost/effectiveness ratio of a CBT for PDA versus a combined treatment of CBT and pharmacotherapy. Eighty-four people followed a CBT either alone or combined with medication. They have been evaluated before treatment, immediately after, as well as one and two years later. The therapy efficacy is calculated at each assessment according to a "Global Functioning Index". Direct and indirect costs are calculated with the "Health Cost Interview". Results suggest that both treatments are equally effective. However, CBT alone is less expensive than CBT with pharmacotherapy. Thus, it offers a better cost-effectiveness ratio that even increases in the long term. 


\section{RESUMEN}

Análisis de costos y de la eficacia de una psicoterapia para el trastorno de pánico con agorafobia en comparación con un tratamiento que combina la farmacoterapia y la psicoterapia.

La farmacoterapia y el tratamiento cognitivo comportamental (TCC) son tratamientos electivos para el trastorno de pánico con agorafobia (TPA) pero generalmente son costosos. El presente estudio busca comparar las proporciones costos/eficacia de un TCC para el TPA con un tratamiento que combina el TCC con una farmacoterapia. Ochenta y cuatro participantes siguieron un TCC combinado o no con medicación. Fueron evaluados antes y después del tratamiento así como uno y dos años después. La eficacia de la terapia se calcula a partir de un "Indice de funcionamiento global" para cada tiempo de medida. Los costos directos e indirectos, por su parte, son calculados gracias a una "Entrevista para determinar los costos de la salud". Los resultados sugieren que para una eficacia equivalente, el TCC sólo es menos costoso que un TCC combinado con la farmacoterapia. Éste ofrece entonces una mejor proporción costos/eficacia. Esta proporción tiende incluso a aumentar con el tiempo.

\section{RESUMO}

\section{Análise dos custos e da eficácia de uma psicoterapia para o Transtorno do Pânico com Agorafobia em comparação com um tratamento que associa a farmacoterapia e a psicoterapia}

A farmacoterapia e a Terapia Cognitivo-Comportamental (TCC) são bons tratamentos para o Transtorno do Pânico com Agorafobia (TPA), mas são, em geral, dispendiosos. O presente estudo visa comparar as relações de custo e eficácia de uma TCC para o TPA em comparação com um tratamento que associa a TCC a uma farmacoterapia. 84 participantes seguiram uma TCC associada ou não a uma medicação. Eles foram avaliados antes e após o tratamento e também depois de um ano e depois de dois anos. A eficácia da terapia foi calculada a partir de um "índice de funcionamento global" para cada tempo de medida. Os custos diretos e indiretos por outro lado, foram calculados graças a uma "entrevista sobre os custos da saúde". Os resultados sugerem que, para uma eficácia equivalente, a TCC sozinha continua sendo a menos dispendiosa que uma TCC associada com uma farmacoterapia. Ela oferece, então, uma melhor relação de custo e eficácia. Esta relação tende até a aumentar com o tempo. 